\title{
Giant cell tumor of the tendon sheath originating from the ankle capsule: A case report and literature review
}

\author{
YU CHEN, XIU-CHUN YU, SONG-FENG XU and BING WANG \\ Department of Orthopaedic Surgery, General Hospital of Jinan Military Region, Jinan, Shandong 250031, P.R. China
}

Received February 13, 2015; Accepted January 18, 2016

DOI: $10.3892 / \mathrm{ol} .2016 .4377$

\begin{abstract}
Giant cell tumor of the tendon sheath (GCTTS), also termed tendosynovial giant cell tumor, is a benign, slow-growing tumor that originates from the tendon sheath or bursa. GCTTS of the foot and ankle is much less frequently reported compared with GCTTS of the hand and knee. However, GCTTS should be considered as a differential diagnosis of soft tissue tumors of the foot and ankle. The optimal treatment strategy for GCTTS in the foot and ankle is controversial due to a scarcity of cases. The present study reports the case of a patient that presented with localized intra-articular GCTTS originating from the capsule of the ankle, which is a rare anatomical location for this tumor. Considering the proximity of the tumor to the adjacent non-tumorous structures, a less radical but complete resection of the tumor was performed, followed by a hydrogen peroxide lavage. There was no evidence of recurrence during a follow-up period of 12 months, and adjuvant radiotherapy was not administered to the patient. A pre-operative diagnosis for GCTTS in the foot and ankle is mainly based on the findings of clinical examination and magnetic resonance imaging, which also facilitates the determination of a surgical strategy. For a localized tumor, an integral resection, as opposed to a radical resection, with a hydrogen peroxide lavage may result in a favorable prognosis. However, the optimal treatment for diffuse GCTTS remains to be identified.
\end{abstract}

\section{Introduction}

Giant cell tumor of the tendon sheath (GCTTS), also termed tendosynovial giant cell tumor, is a benign, slow-growing tumor that originates from the tendon sheath or bursa (1). GCTTS is a type of tumor that mainly occurs in the hand, and a previous study reported that only $3-5 \%$ of GCTTS are detected

Correspondence to: Professor Xiu-Chun Yu, Department of Orthopaedic Surgery, General Hospital of Jinan Military Region, 25 Shifan Road, Jinan, Shandong 250031, P.R. China

E-mail: toeflmugou@163.com

Key words: tendosynovial giant cell tumor, giant cell tumor of tendon sheath, foot and ankle, surgery in the foot and ankle (1). Microscopically, GCTTS is characterized by a mixture of multinuclear giant cells, mononuclear cells and histiocytes $(1,2)$. GCTTS typically arises from the flexor tendon sheath of the hand, but GCTTS in the foot is much less frequently reported (3). However, GCTTS should be considered as a differential diagnosis of soft tissue tumors observed in the foot and ankle. Literature regarding treatment strategies of GCTTS in the foot and ankle are limited due to a scarcity of patients with this tumor type (Table I). Clinicians generally refer to personal experience and preference when choosing surgical protocols from various alternative operations for GCTTS patients. However, the improper selection of surgical treatment may lead to unfavorable outcomes and an unexpected burden on the patient; hence, additional analyses of cases that have been diagnosed with GCTTS in the foot and ankle are warranted for better postoperative outcomes for patients. The present study describes the case of a patient presenting with intra-articular GCTTS arising from the right ankle capsule, which is a rare anatomical location for this tumor.

\section{Case report}

A 58-year-old female patient was referred to the General Hospital of Jinan Military Region (Jinan, Shandong, China) in December 2012 with a slow-growing swelling in the anterior aspect of the right ankle that had been developing over 12 months. The patient felt mild pain in the swelling a week prior to hospital admission, but there was no history of trauma to the region prior to the onset of symptoms. A clinical examination revealed the presence of a $3.0 \times 4.0 \mathrm{~cm}$ non-tender firm tumor in the anterior aspect of the right ankle (Fig. 1). There was no free mobility of the tumor over the underlying bone surface, and the range of motion of the right ankle was not compromised. A sonography revealed that the tumor possessed a solid, homogeneous and hypoechoic nature. Magnetic resonance imaging (MRI) indicated that the tumor was located in front of the tibiotalar joint surface with isointensity on T1 weighted imaging and mild hyperintensity on T2 weighted imaging. There was no significant osseous erosion in the adjoining bone (Fig. 2). A marginal excision of the tumor was performed under epidural anesthesia. Intra-operatively, the tumor was well-circumscribed and gray-yellow, and was observed to originate from the ankle capsule. Dorsalis pedis vessels and a superficial peroneal nerve were dislocated laterally due to 


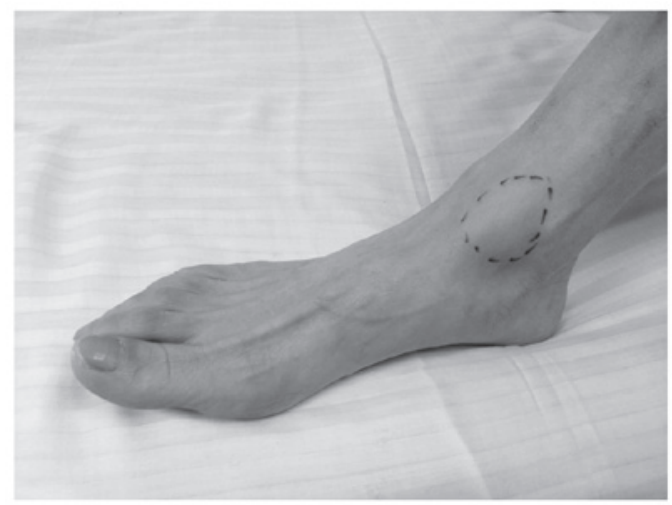

Figure 1. Patient presenting with a tendosynovial giant cell tumor in the right ankle capsule.

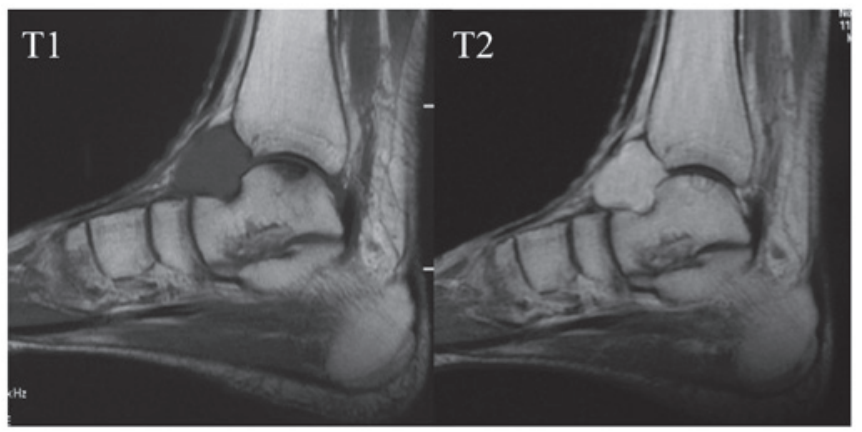

Figure 2. Magnetic resonance imaging of the tumor. The tumor was located in front of the tibiotalar joint surface, with isointensity on the T1 weighted image and mild hyperintensity on the $\mathrm{T} 2$ weighted image.

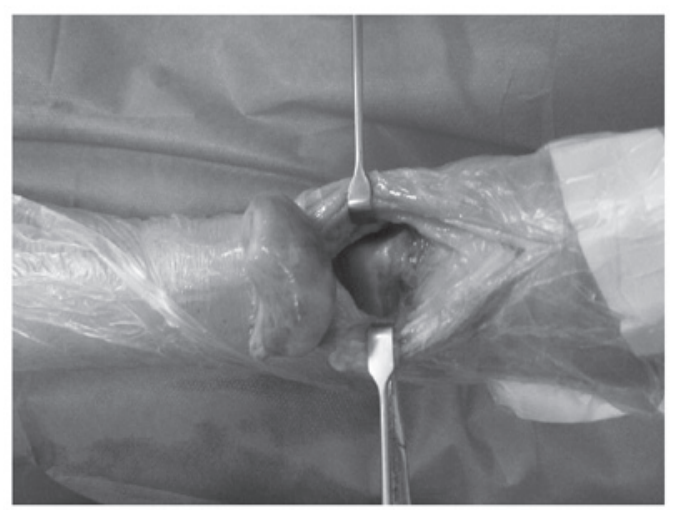

Figure 3. Intraoperative findings. A marginal excision of the tumor was performed under epidural anesthesia. The tumor originated from the ankle capsule. Dorsalis pedis vessels and a superficial peroneal nerve were dislocated laterally due to compression by the tumor.

compression by the tumor. The marginal resection of the tumor was followed by a hydrogen peroxide lavage. The tumor was not malignant and the adjacent non-tumorous structures were preserved. An imprint in the front aspect of the underlying tibiotalar joint was observed, due to the lasting compression by the tumor (Fig. 3). The capsule of the ankle was sutured and the ankle was immobilized for 3 weeks post-operatively.

The resected tumor was sent to the Department of Pathology, General Hospital of Jinan Military Region, for

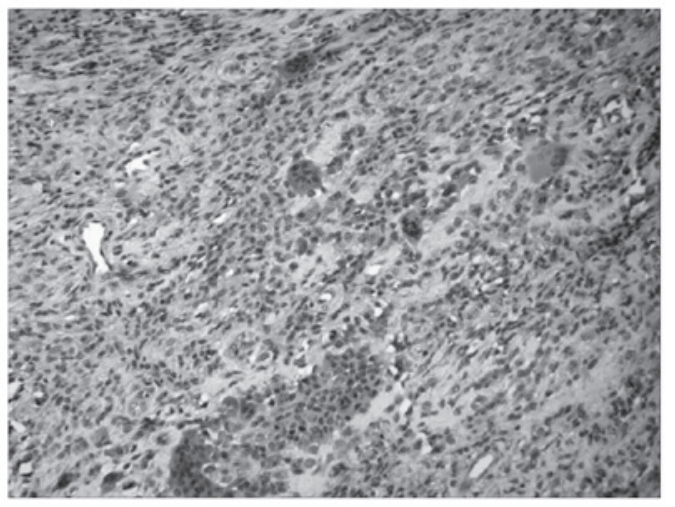

Figure 4. Typical microscopic features of a giant cell tumor of the tendon sheath, with a mixture of mononucleated cells and multinuclear cells and thick fibrous septae. Hematoxylin-eosin staining; magnification, x200.

histological analysis. Histological staining with hematoxylin and eosin revealed a well-encapsulated tumor composed of a mixture of mononucleated cells and multinuclear cells with thick fibrous septae (Fig. 4). The patient was subsequently diagnosed with GCTTS. Due to the lack of involvement of adjacent structures, no adjuvant therapy was provided to the patient. There was no evidence of recurrence during a follow-up period of 12 months.

\section{Discussion}

GCTTS, also termed tendosynovial giant cell tumor, is a benign, slow-growing solid soft-tissue tumor, with a mild female predilection $(4,5)$. The most common clinical observation is the development of an asymptomatic mass, and additional symptoms, including pain and a limited range of motion in the joints, are suggestive of compression or involvement of structures adjacent to the tumor $(3,4)$. The origins of GCTTS remain to be elucidated, including whether the tumor is neoplastic or reactive. Karyotype analysis has identified abnormal cells in GCTTS, which is considered to be evidence of neoplasia, whereas polymerase chain reaction-based assays for methylation of the X-linked human androgen receptor gene suggest GCTTS develops from polyclonal proliferation, indicating that the disease is reactive or hyperplastic (6). In addition, GCTTS is considered to be due to injury, indicating a reactive nature; however, this association has not been determined (7). Therefore, the nature of GCTTS remains to be elucidated.

The involvement of the forefoot and hindfoot are common locations of GCTTS of the foot and ankle, and the tumors are primarily reported to originate from tendon sheaths surrounding the ankle $(1,8)$. In the present patient, the localized tumor originated from the capsule of the ankle, and the patient presented with an intra-articular mass, which is extremely rare for GCTTS in this anatomical region. Although GCTTS rarely involves the foot and ankle, it is extremely important to consider GCTTS as a differential diagnosis for soft tissue tumors in this location. In addition to GCTTS, differential diagnosis of soft tissue tumors in the foot and ankle may consist of lipoma, a synovial cyst, pigmented villondular synovitis (PVNS), fibromatosis, undifferentiated pleomophic sarcoma, 


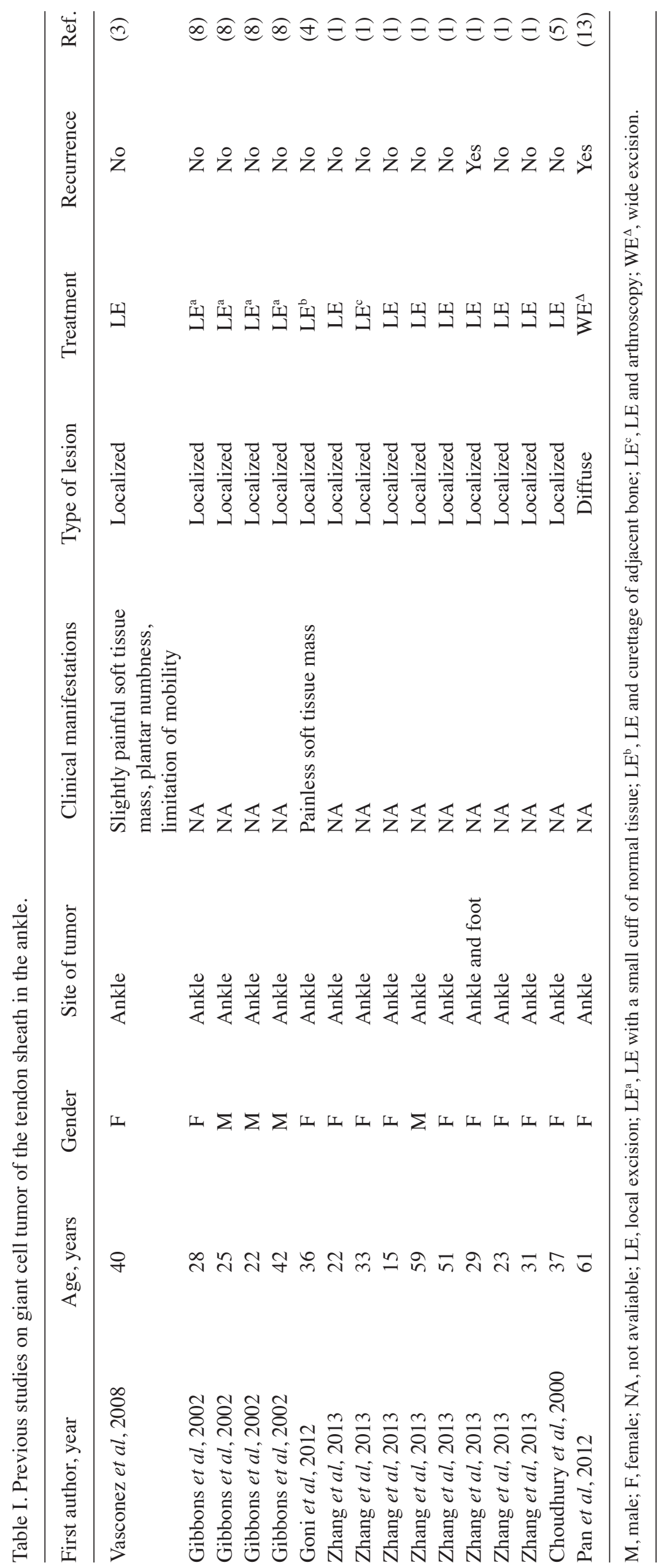


synovial sarcoma and leiomyosarcoma $(4,9)$. Imaging, such as ultrasonography and MRI, has been identified as effective in differentiating GCTTS from other soft tissue tumors in the foot and ankle (5). Ultrasonography differentiates a solid mass from a cyst, and sonographically, GCTTS is a typically homogeneous, solid and hypoechoic mass (10). Resonance imaging is the most valuable modality in identifying GCTTS in the foot and ankle. MRI features of GCTTS are associated with hemosiderin content in the tumor, and tumors typically exhibit iso- or hypointensity on T1 weighted images and hypointensity on T2 weighted images (11). Notably, the MRI features of GCTTS are similar to those of PVNS, particularly when GCTTS is diffuse-type (1). GCTTS originates from the extra-articular synovium, and as a result, the majority of lesions are located outside the ankle capsule, even when intra-articular structures are involved (12). By contrast, PVNS arises from the intra-articular synovium and primarily involves intra-articular osseous and chondral structures $(1,13)$. Patients present with limited motion in the ankle at an early-stage of disease $(1,13)$. A precise pre-operative diagnosis must be determined since the surgical treatment of the two tumors is different; a local resection with the removal of affected tissue is reported to be effective in treating GCTTS, whereas a wide excision with ankle fusion is recommend in PVNS, since the articular cartilage and subchondral bone are extensively eroded $(1,13)$.

Studies regarding treatment of GCCTS in the foot and ankle are rare due to the scarcity of the tumor (Table I). The recurrence rate of GCTTS has been reported to be as high as $44 \%$ and an integral removal of the tumor is recommended as the pivotal treatment to prevent local recurrence (12). In the present study, the patient presented with a localized intra-articular GCTTS of the ankle capsule, and the lesion was integrally removed followed by a hydrogen peroxide lavage. The capsule was preserved for the reconstruction of the ankle capsule and radical resection was avoided due to the proximity of the tumor to the adjacent non-tumorous structures. There was no evidence of recurrence during follow-up. The present findings suggest that a local excision of localized GCTTS in the ankle may be the treatment of choice, since the removal of adjacent structures may be impossible. The optimal treatment strategy regarding diffuse GCTTS, with involvement of adjacent structures, including bone and cartilage, is controversial and the recurrence rate in this type of GCTTS is increased (1). Pan et al (13) reviewed the cases of patients with GCTTS in the lower extremities and the authors suggested that the articular structures should be exposed extensively in patients with diffuse GCTTS and the affected structures require integral excision. In addition, the authors advised that adjuvant radiotherapy treatment is provided to patients to prevent local recurrence. Goda et al (14) conducted radiotherapy treatment for localized GCTTS in the thumb with a customized device, and the outcome of this was favorable. The present study hypothesizes that the prognosis of patients presenting with GCTTS of the foot and ankle is dependent on a precise pre-operative diagnosis and optimal treatment strategy based on the biological behaviors of the tumors; however, the efficacy of adjuvant therapy is unclear. The treatment of GCTTS, particularly in diffuse GCTTS, remains to be determined, and therefore additional clinical studies are required.

\section{References}

1. Zhang Y, Huang J, Ma X, Wang X, Zhang C and Chen L: Giant cell tumor of the tendon sheath in the foot and ankle: Case series and review of the literature. J Foot Ankle Surg 52: 24-27, 2013.

2. Ushijima M, Hashimoto H, Tsuneyoshi M and Enjoji M: Giant cell tumor of the tendon sheath (nodular tenosynovitis). A study of 207 cases to compare the large joint group with the common digit group. Cancer 57: 875-884, 1986.

3. Vasconez HC, Nisanci M and Lee EY: Giant cell tumour of the flexor tendon sheath of the foot. J Plast Reconstr Aesthet Surg 61: 815-818, 2008.

4. Goni V, Gopinathan NR, Radotra BD, Viswanathan VK, Logithasan RK and Balaji S: Giant cell tumour of peroneus brevis tendon sheath - a case report and review of literature. BMJ Case Rep 2012: 2012.

5. Choudhury M, Jain R, Nangia A and Logani KB: Localized tenosynovial giant cell tumor of tendon sheath. A case report. Acta Cytol 44: 463-466, 2000.

6. Vogrincic GS, O'Connell JX and Gilks CB: Giant cell tumor of tendon sheath is a polyclonal cellular proliferation. Hum Pathol 28: 815-819, 1997.

7. Monaghan H, Salter DM and Al-Nafussi A: Giant cell tumour of tendon sheath (localised nodular tenosynovitis): Clinicopathological features of 71 cases. J Clin Pathol 54: 404-407, 2001

8. Gibbons CL, Khwaja HA, Cole AS, Cooke PH and Athanasou NA: Giant-cell tumour of the tendon sheath in the foot and ankle. J Bone Joint Surg Br 84: 1000-1003, 2002.

9. Bancroft LW, Peterson JJ and Kransdorf MJ: Imaging of soft tissue lesions of the foot and ankle. Radiol Clin North Am 46: 1093-1103, vii, 2008.

10. Middleton WD, Patel V, Teefey SA and Boyer MI: Giant cell tumors of the tendon sheath: Analysis of sonographic findings. AJR Am J Roentgenol 183: 337-339, 2004.

11. De Beuckeleer L, De Schepper A, De Belder F, Van Goethem J, Marques MC, Broeckx J, Verstraete K and Vermaut F: Magnetic resonance imaging of localized giant cell tumour of the tendon sheath (MRI of localized GCTTS). Eur Radiol 7: 198-201, 1997.

12. Reilly KE, Stern PJ and Dale JA: Recurrent giant cell tumors of the tendon sheath. J Hand Surg Am 24: 1298-1302, 1999.

13. Pan GP, Zhao LJ, Fang Y and Feng RH: Diagnosis and clinical application of MRI for giant cell tumor of tendon sheath in lower extremity. Zhongguo Gu Shang 25: 953-956, 2012 (In Chinese).

14. Goda JS, Patil P, Krishnappan C and Elangovan D: Giant cell tumor of the tendon sheath treated by brachytherapy (surface mold) technique-A technical illustration. Brachytherapy 8: 79-83, 2009. 